\title{
Variations
}

Variations

Revue internationale de théorie critique

15 | 2011

La haine

\section{Au sujet de la vie mutilée}

Précarité, flexibilité du travail, discours sécuritaires

\section{Alexander Neumann}

\section{(2) OpenEdition}

Journals

\section{Édition électronique}

URL : http://journals.openedition.org/variations/103

DOI : 10.4000/variations. 103

ISSN : 1968-3960

Éditeur

Les amis de Variations

Édition imprimée

Date de publication : 1 mars 2011

Référence électronique

Alexander Neumann, «Au sujet de la vie mutilée », Variations [En ligne], 15 | 2011, mis en ligne le 01 février 2012, consulté le 30 avril 2019. URL : http://journals.openedition.org/variations/103 ; DOI : 10.4000/variations. 103 


\section{Alexander Neumann}

\section{Au sujet de la vie mutilée}

\section{Précarité, flexibilité du travail, discours sécuritaires}

La crise globale a mis en lumière la relation qui se noue entre l'insécurité sociale et l'espace public bourgeois, l'expérience vécue des citoyens et la politique. Cela apparait en France dans la réception publique des suicides du travail, la préoccupation massive du chômage, le conflit sur les retraites de septembre-octobre 2011. Sur le plan politique ce nœud se serre dans le déclenchement de crises politiques majeures en Europe sur fond de mouvements de grève comme par exemple au Portugal en 2011. Simultanément, la crise favorise la percée de l'extrême droite européenne, amorcée avant la crise de 2008, qui s'amplifie depuis avec sa participation gouvernementale dans de nombreux pays membre, en Italie, aux Pays Bas, au Danemark...

Ce lien entre la précarisation sociale et la contestation politique, qui apparaît comme une réalité palpable, empiriquement saisissable, est précisément impensable avec les notions du mainstream des sciences sociales, issu des années 1980. L'une des figures les plus éminentes et les plus respectables de cette théorisation est Jürgen Habermas avec une orientation qui sépare fondamentalement le travail et l'action, le marché et la politique, ou encore l'expérience vécue des citoyens et la crise de l'espace public bourgeois.

Le présent texte entend saisir les effets publics de l'insécurité sociale, à travers la conjugaison d'apports critiques de la sociologie du travail et de la sociologie politique. En effet, les deux disciplines sont le plus souvent considérées comme des champs distincts sans relation de 
réciprocité théorique, éventuellement complémentaires, mais dans un rapport d'extériorité l'un à l'autre. Pourtant, la notion même de «société du risque » qui a marqué de son empreinte la sociologie européenne ces vingt dernières années ${ }^{1}$ nécessite une telle mise en relation, si l'on ne veut pas se contenter d'un discours superficiel sur le phénomène de l'insécurité sociale. Dans son livre désormais classique, Beck décrit précisément les prémisses de la flexibilité du travail et de la précarité, aujourd'hui en voie de généralisation. L'auteur en déduisait alors que les structures fondamentales de la société subissaient des changements qui affectent ses principes d'organisation, donc son avenir politique. Des représentants de la Théorie critique reprochent à Beck de ne pas argumenter cette constellation de façon précise, mais non pas de l'avoir soulevée ${ }^{2}$.

Dans un premier temps, je vais aborder les aspects empiriques de l'insécurité sociale en Europe, en soulignant notamment l'interdépendance de la pauvreté, de la précarité et de la flexibilité. Sur cette lancée, nous préciserons en quoi la flexibilité du travail doit être considérée comme une partie intégrante de l'insécurité sociale. Ensuite, nous allons cerner les effets de ces dimensions de l'insécurité sur la communication sociale et les structures de la personnalité qui la fondent, afin de comprendre l'érosion des espaces publics. Enfin, nous verrons comment les institutions européennes entendent répondre à cette situation, à travers des dispositifs sécuritaires (Flexicurity et dispositifs de sécurité publique).

\section{L'insécurité sociale dans l'Union européenne}

L'ensemble des études sociologiques, des rapports d'enquête et des statistiques européennes existantes, indique que les manifestations de l'insécurité sociale sont en progression en Europe

\footnotetext{
$1 \quad$ Beck, Ulrich (1981), Die Risikogesellschaft, Suhrkamp, Frankfurt/M. ; Panzer, Gerhard (2001), Kairos der Risikogesellschaft, Kassel University Press.

${ }^{2}$ Negt (2001), Arbeit und menschliche Würde, Steidl, Göttingen.
} 
depuis des années. Cela bien avant le déclenchement de la crise globale en 2008. Aujourd'hui, 10\% de la population européenne qui est obligé de travailler pour vivre est officiellement considérée comme étant au chômage, selon Eurostat (2010). Ces statistiques, qui écartent par définition les travailleurs sans papiers, ne concernent que les personnes qui peuvent postuler à tout moment à un emploi à temps plein. Cela écarte par définition tout l'éventail des demandeurs à temps partiel, des bénéficiaires de minimas sociaux qui passent par des petits boulots, les radiés, etc. La statistique européenne, déjà spectaculaire dans ses résultats, ne désigne donc que la point d'un iceberg de situations de précarité, de pauvreté avec ou sans emploi, et qui correspondent à des situations de travail dites stables souvent tendues ou angoissantes dans les grandes entreprises de type France Telecom, Renault ou encore IBM. La moyenne de 10\% de chômeurs varie elle-même fortement, avec des piques en Espagne ou parmi les jeunes citoyens peu qualifiés en France, atteignant 40\% par endroits, et des lieux moins marqués, comme parmi les salariés qualifiés des grandes villes du Sud ou de l'Ouest de l'Allemagne.

Cette ampleur de la précarisation accrédite le sentiment des citoyens que les différents aspects du problème soient liées, par exemple lorsqu'un Français sur deux déclare d'avoir peur de finir comme Sans domicile fixe (dans un sondage BVA de décembre 2006 et d'autres qui ont suivi en 2008). Même les classes moyennes se sentent donc concernées par les tendances à la précarisation. Bien avant la grande crise de 2008, les enquêtes sociologiques ont souligné que la précarité du travail conduit souvent à une grande vulnérabilité sociale et à la marginalisation ${ }^{1}$. En Allemagne, les études disponibles au sujet des publics concernés par les lois Hartz (gestion des chômeurs et de l'aide sociale) montrent une tendance à la relégation vers la pauvreté, et un lien entre l'introduction des nouvelles normes de gestion et l'accroissement de la pauvreté des enfants (Buntenbach, 2007). Cette démonstration pourrait être répétée pour chaque pays de l'Union

Paugam, Serge (2004), Le salarié de la précarité, PUF, Paris. 
européenne, à quelques variations nationales près. La Commission européenne souligne à son tour l'accroissement de l'emploi et des contrats flexibles ou précaires dans l'Europe actuelle ${ }^{1}$. La crise économique mondiale ne fait qu'accélérer ces tendances lourdes.

La précarité de l'emploi, sous la forme de stages, de contrats courts, intérimaires ou peu protégés, est elle aussi en expansion constante.

La Grande Bretagne est l'un des pays où l'évolution vers des contrats précaires a pris une forme très nette depuis les années $1980^{2}$. Cependant, des sociétés jadis réputées pour leur opposition au capitalisme flexible, comme la France ou l'Allemagne, sont aujourd'hui engagées dans des processus similaires ${ }^{3}$.

En France, au sein d'une population active d'environ 27 millions de personnes, à peu près un tiers échappe au travail régulier à temps plein : Chômeurs, contrats précaires ou à temps partiel, intérim, stages, etc ${ }^{4}$. Cette proportion a explosé depuis les années 1980 : Le nombre de contrats en temps partiel est passé de 15000 à 1.860000 (entre 1983 et 2005), alors que le nombre de CDD est passé de 260000 à 1.600000.

En Allemagne, un tiers des 27 millions de salariés était concerné par des situations contractuelles précaires $^{5}$. Le nombre des postes en temps partiel est monté à 12 millions et le nombre de CDD à presque deux millions.

Cette évolution implique des changements qualitatifs. $85 \%$ des salariés français en CDD déclarent que cela les « empêche de faire des projets à long terme. ${ }^{6}$ "

\footnotetext{
Commission of the European Communities (11/2006), Green Paper : Modernising labor law to meet the challenges of the 21th century, Brussels., p. 8

2 Mc Govern, P. et alli, (2004), «Bad Jobs in Britain. Nonstandard Employment and Job Quality » in : Work and Occupations N.31, pp. 225-249.

Pfefferkorn, Roland (2007), Inégalités et rapports sociaux, La Dispute, Paris.

Neumann (2005), « Conscience de casse » in Variations n.6, éditions Parangon, Lyon.

Ibid., p. 127

6 Dares, «Contrats à durée déterminée, intérim, apprentissage, contrats aidés. Les emplois à statut particulier ont progressé entre 1982 et 2002 », Premières synthèses, 4/2005.
} 
Le changement le plus significatif est sans doute l'apparition de la catégorie des Working poor, ces salariés pauvres qui occupent un emploi ${ }^{1}$.

La précarité de l'emploi trouve une prolongation dans la peur des salariés bien intégrés du privé de perdre leur poste ou d'être sanctionnés s'ils ne répondent pas aux normes de gestion des entreprises. Cela explique une autre forme d'insécurité sociale, liée au stress dans le travail, à l'intensification du travail et à l'accroissement de la flexibilité ${ }^{2}$ (Mc Govern et alli, 2007 ; Paugam, 2000).

La flexibilité des horaires et des modes de gestion du travail peut ainsi être cernée comme étant une facette du processus général de l'insécurité sociale. La peur de ne pas répondre aux nouvelles normes de travail, la déconstruction des collectifs de travail par le management par projet et la focalisation des activités sur le court terme sont quelques-unes des caractéristiques de ce type d'insécurité. La flexibilité dans les entreprises correspond par ailleurs à l'usage d'une main d'œuvre externe qui est souvent précaire. Les différents aspects de l'insécurité sociale (dans le travail, la précarité ou la pauvreté) se combinent et interagissent.

\section{La flexibilité comme forme d'insécurité sociale}

La flexibilité est généralement associée au management performant des grandes entreprises, renvoyant ainsi à l'idée d'un salariat bien intégré et protégé. Nous allons pourtant montrer que la flexibilité du travail est au contraire une face complémentaire de l'insécurité sociale.

Bundesregierung der Bundesrepublik Deutschland (2005), Der 2. Armutsbericht, Berlin.

Mc Govern, P. et alli, (2004), «Bad Jobs in Britain. Nonstandard Employment and Job Quality » in : Work and Occupations N.31, pp. 225-249. 
La flexibilité du travail prend son essor dans la dissociation du temps de production et du temps de travail individuel. Cette césure nous paraît fondamentale pour comprendre les nouvelles formes de l'organisation du travail, qui ne sont plus entravées par les rythmes de la vie quotidienne des salariés, mais qui répondent aux rythmes de développement de l'entreprise et des marchés. Les ruptures potentielles qui sont engendrées par la dissociation des temps de production et des temps de travail ont été soulignées à différents moments par des sociologues critiques en France, en Allemagne et en Grande Bretagne ${ }^{1}$.

Au sein du nouveau cadre, la distinction sociologique, entre une flexibilité « interne » à l'entreprise et, une autre « externe » (faisant appel à l'Intérim et des contrats précaires) perd sa signification. Nous pouvons avancer une série d'aspects empiriques qui illustrent l'abolition des frontières entre le champ « interne » et « externe ». La gestion flexible des personnels des grandes entreprises et l'appel à des contrats précaires non seulement se complètent, mais s'enchevêtrent le plus souvent.

Nous allons successivement aborder l'entrée des contrats et situations précaires au cœur de la gestion des grandes entreprises, puis indiquer les effets de cette flexibilité sur le marché du travail.

Le management flexible et différencié du temps de travail s'est appuyé sur des structures hiérarchiques fortes et maintenues ${ }^{2}$, mais la complexité des nouveaux rythmes de travail exige une responsabilisation des salariés dans l'organisation des horaires. Cette situation éclaire le phénomène de «l'autonomie » partielle que le management des grandes entreprises accorde souvent pour la répartition des horaires au sein des équipes de travail ou des groupes de projet. Comme nous l'avons

\footnotetext{
Naville, Pierre, (1964), La classe ouvrière et le régime gaulliste, EDI, Paris ; Vincent, Jean-Marie (2001), Un autre Marx. Après les marxismes, éd. Page 2, Lausanne; Negt (2001), Arbeit und menschliche Würde, Steidl, Göttingen. Voir aussi A.Neumann, (2010) Kritische Arbeitssoziologie, ed. Schmetterling, Stuttgart.

2 Linhart, Danièle (1994), La modernisation des entreprises, La Découverte, Paris ; Coutraut, Thomas (1998), L'entreprise néolibérale, La Découverte, Paris.
} 
montré pour les cadres français, la flexibilisation du temps de travail accroît la liberté de gestion des tâches et des heures de présence au travail, tout en imposant des contraintes temporelles sous la forme d'un rythme de travail plus soutenu ou plus irrégulier. Un responsable syndical allemand souligne que le nouveau mot d'ordre managérial s'appliquant à cette situation a été parfaitement résumé par la DRH d'IBM : «Faites ce que vous voulez, mais faites du profit ! ${ }^{1} »$

Cette autonomie proclamée se heurte non seulement aux contraintes exercées par le mouvement du marché, mais également par le maintien d'un contrôle managérial assez étroit sur le processus du travail : «Tout le problème de l'organisation est de conserver un contrôle suffisamment serré sur les activités, sur les décisions, pour éviter des marchandages qui pourraient lui échapper. Le management de projet, parce qu'il disperse les personnes, conduit par conséquent à la centralisation des décisions-clés concernant le projet... Rien ne doit échapper aux gouvernants ${ }^{2} . »$

La maîtrise du temps se situe également du côté du management central de l'entreprise. Tandis que le « gouvernement » de l'entreprise structure le temps long des décisions stratégiques, de la programmation pluriannuelle, de l'allocation des budgets et des « ressources humaines », le « projet » n'existe qu'à court terme. L'expérience concrète des salariés se limite à ce temps court, qui peut varier de quelques semaines à plusieurs mois ou plus rarement un an. À l'intérieur du projet, le délai, qui lui sert de cadre, impose des références temporelles réduites : la gestion immédiate, l'urgence, l'accélération et le « bouclage ». Comme la projection dans le temps diminue considérablement, le rapport à l'autre devient plus instrumental, plus proche de l'arrangement que de l'entendement. Le stress est inscrit dans la temporalité du projet ${ }^{3}$.

$1 \quad$ Pickshaus/Urban (2001), Arbeiten ohne Ende. Neue Arbeitsverhältnisse und gewerkschaftliche Arbeitspolitik, ed. VSA, Hamburg, p. 13

2 David Courpasson, L'action contrainte, PUF, 2000, p.210.

3 Pickshaus/Urban (2001), Arbeiten ohne Ende. Neue Arbeitsverhältnisse und gewerkschaftliche Arbeitspolitik, ed. 
L'insécurité et l'incertitude sociales sont donc une partie intégrantes de la gestion flexible des entreprises, et ne présentent pas des phénomènes annexes ou extérieurs.

Même si les modalités de gestion flexible du temps de travail divergent fortement selon le degré de qualification des salariés, la centralité des rythmes du marché pour l'organisation du travail -quotidien, hebdomadaire et annuel- apparaît comme une donnée transversale dans le management des entreprises : "Il s'agit du noyau dur des stratégies de rationalisation actuelles et qui concerne aussi bien l'ingénieur en recherche et développement que l'ouvrière en équipe du secteur automobile. $\left.{ }^{l}\right\rangle$

\section{3. Érosion structurelle et distorsion communicationnelle}

Dans ce contexte flexible et précaire, l'expression publique des salariés ne passe plus par la représentation d'une classe ouvrière industrielle, qualifiée et masculine. Le mode de communication sociale même qu'organisait la classe ouvrière au sein du salariat a volé en éclat. L'un des fondements de la participation publique qui s'organisait par ce cadre, non sans restrictions, était la régularité du temps social, qui donnait le rythme de l'expérience collective : «L'ancienne réglementation du temps de travail avait favorisé la création de collectifs ouvriers dans les entreprises, par la contrainte commune, qui servait de fondement à la communication et à la formation de la solidarité... L'imaginaire social des ouvriers était alors caractérisé par une conscience dichotomique: "nous en bas, vous en haut». Le destin commun et le travail corporel étaient les traits caractéristiques de cette identité de soi. ${ }^{2} \gg$

Cette structure de communication, qui était marquée par la contrainte collective, permettait la formulation de souhaits collectifs, à défaut d'exprimer les souhaits d'autonomie de chaque

VSA, Hamburg, p. 61

$1 \quad$ Lehndorff, Steffen (2000), Wiederaneignung der Zeit, VSA, Hamburg, p. 26

2 Negt (2001), Arbeit und menschliche Würde, Steidl, Göttingen, p. 198 
salarié. Nous avons vu que les revendications d'autonomie, dans le sens d'une émancipation des contraintes du travail, ont contribué à déconstruire l'ancienne culture ouvrière, dès les années 1960 . La critique du travail et de l'éthique de métier protestante ont précisément constitué l'un des éléments du changement des dispositifs managériaux ${ }^{1}$.

L'individualisation des situations contractuelles et la flexibilisation des temporalités du travail achèvent la désorganisation des structures de communication traditionnelles, tandis que les nouvelles formes de délibération des salariés sont loin d'être généralisées. Nous pensons notamment aux groupes d'expression indépendants, aux assemblées de débat sur les expériences de la flexibilité, aux collectifs de précaires et aux associations de chômeurs.

Pour le moment, la déstructuration de l'expérience commune et de la prise de parole des salariés semble prévaloir. Dans les grandes entreprises, les temps de discussion sont écourtés, sinon supprimés dans le cadre de la flexibilité, alors que les équipes et les individus changent sans cesse. Ils n'ont à peine le temps de construire des rapports de confiance et de communication horizontale. La flexibilité du temps empêche précisément l'échange entre salariés au sujet de l'expérience vécue de la flexibilité. Le temps partiel et les contrats à durée indéterminée contribuent également à démembrer les rythmes communs aux salariés, rendant plus difficile la prise de parole et la discussion collective.

Ces processus ont de multiples incidences sur la structuration et la vie de l'espace public. En même temps que la communication horizontale est rendue encore plus difficile qu'elle ne l'était dans le cadre des entreprises fordistes, le management déploie activement son propre espace de communication, que Negt appelle l'espace public de production. Il ne s'agit plus seulement de la propagande interne et du travail de relations publiques, mais d'une approche visant l'adhésion directe au discours managérial. De grandes assemblées de présentation, associant salariés et cadres,

Boltanski / Chiappello (1999), Le nouvel esprit du capitalisme, Gallimard, Paris. 
sont organisées pour faire partager le projet de l'entreprise, aussi bien dans le secteur privé que public. Dans le privé, le recours au référendum à l'initiative du management peut servir à légitimer des décisions discutables, par exemple l'allongement de la durée du travail ou l'amplification de la flexibilité (pratiquée par de nombreuses entreprises tel Bosch, Siemens, plusieurs firmes de l'automobile, etc.). Bien qu'il s'agisse de formes publiques - l'assemblée ou le référendum - on assiste au fond à des représentations privées dénuées de débat contradictoire, qu'Habermas désigne comme étant de la «publicité manipulatoire déployée d'en haut », d'autant plus qu'elle se déroule dans la sphère privée de l'entreprise. Les représentations semi-privées de la flexibilité, ainsi que la publicité plébiscitaire ou manipulatoire dont parle Habermas, tendent à empêcher une délibération portant sur les souhaits des salariés.

Dans le même temps, les mass-media et l'industrie de la culture neutralisent une grande partie du temps libre. Le démembrement flexible des temporalités sociales, et la subordination du temps libre à la sphère privée, s’enchevêtrent ainsi : «La pénétration des médias dans la sphère privée, qui favorise le divertissement et la dispersion de l'imaginaire individuel, est anticipée par le découpage de la force de travail dans les différents domaines de la production. ' $\gg$ Pendant ce temps, syndicats et partis de masse contribuent majoritairement à reproduire les anciennes structures organisationnelles, devenues inopérantes pour la communication entre salariés.

L'ensemble des dimensions de l'insécurité sociale et de la flexibilité décrivent une érosion structurelle des dispositifs hérités de l'Etat Providence, qui provoque des réactions défensives individuelles et collectives parfois très fortes, mais dont la traduction politique est singulièrement faible et aléatoire ${ }^{2}$.

Par conséquent, l'érosion des structures sociales traditionnelles affecte profondément Negt (2001), Arbeit und menschliche Würde, Steidl, Göttingen, p. 199

2 Neumann, (2007), «Le courant chaud de la Théorie critique : Subjectivité rebelle, espace public oppositionnel, travail vivant $»$ in : O. Negt, L'espace public oppositionnel, Payot, Paris. 
l'espace public et les modes de participation. L'un des phénomènes les plus visibles est l'abstention électorale ou les votes de rejet sans projet politique, comme on a pu le constater lors des crises politiques de ces dernières années en France (en 2002 et en 2005), au Pays Bas et en Belgique (en 2007), en Allemagne (en 2006) ou lors du refus du traité de Lisbonne en Irlande (en 2008), et encore l'insurrection de la jeunesse grecque en décembre 2008 .

Pour comprendre pareille interaction, il convient de mettre en relation, d'un côté, le champ privé de l'insécurité sociale et, de l'autre, les effets que ce champ produit dans la sphère de la délibération publique. Les discriminations liés aux origines sociales, l'expérience pratique de la flexibilité ou de la précarité du travail, sont des phénomènes non-publics, mais ils interfèrent néanmoins fortement sur les capacités de communication des citoyens et acteurs, comme nous allons le souligner. La désaffiliation sociale conduit au désistement et au retrait de l'espace public.

D’abord il est possible de rappeler l'étude classique au sujet des «Chômeurs de Marienthal » d'avant la deuxième Guerre Mondiale, qui montre toute l'étendue de la déstructuration sociale et symbolique dont sont frappés les privés d'emploi, ce qui inhibe leur prise de parole et leur capacité de participer à l'espace public. Une observation plus récente, mais similaire a été réalisée au sujet des chômeurs du département du Nord ${ }^{1}$.

Le lien entre le chômage de longue durée, la destructuration de la personnalité et le retrait de l'espace public n'est donc plus à démontrer. Il peut être résumé de façon radicale de la façon suivante : «Le chômage est un acte violent. Il s'agit d'une atteinte à l'intégrité physique et psychique. Le chômage représente le pillage et l'expropriation des facultés et qualités que des personnes ont difficilement acquises à travers un processus de formation, impliquant la famille, l'école et la formation professionnelle².»

Schwarz, Olivier (1990), Le monde privé des ouvriers, PUF, Paris.

Negt, Oskar (2007), L'espace public oppositionnel, Payot et Rivages, Paris, p. 179 
Du point de vue théorique, la déstabilisation de la personnalité entraîne obligatoirement l'incapacité de participer activement à l'espace public, étant donné que la formation de cet espace public dépend de l'existence de personnes autonomes ${ }^{1}$.

Les formes précaires du travail qui ne s'apparentent pas directement au chômage ont été peu analysées dans leur relation avec le désinvestissement public des salariés. Ce n'est pas un simple manque de temps qui explique le retrait ou le repli protestataire des catégories précaires, mais bien davantage une paralysie liée à la peur d'une vie incontrôlable. La peur remplit ici le rôle d'une « réaction défensive » au sens freudien du terme ${ }^{2}$.

Enfin, l'expérience de la flexibilité du travail, de la part de salariés qualifiés et intégrés, peut produire des effets comparables à ceux que provoque la précarité du travail. Cela malgré la distinction sociologique traditionnelle, fortement établie qui tend à identifier le travail à une source d'intégration sociale.

Le processus de la flexibilisation se présente avant tout comme une restructuration perpétuelle, comme une transition prolongée vers une forme sociale incertaine et comme un mouvement corrosif qui ébranle les personnalités et les habitus. À ce propos, Richard Sennet parle de la « corrosion » du caractère social des individus ${ }^{3}$.

Sennett aborde le problème de la déconstruction des structures temporelles et sociales habituelles, lorsqu'il met en évidence l'irruption des jugements et actions à “ court-terme” dans les modes de travail et de vie ${ }^{4}$ Après avoir cité plusieurs exemples de flexibilisation du temps de travail,

Habermas (1990), Strukturwandel der Öffentlichkeit, Suhrkamp, Frankfurt/M.

Neumann (2006), « Pour une écoute sociologique » in : Variations - revue internationale de théorie critique N.8, Ed. Parangon, Lyon.

Richard Sennett (2000), Le travail sans qualité, 10/18, Paris.

$4 \quad$ Op.cit, p.26. 
tel l'intérim, la mobilité flexible des cadres, etc., il formule un diagnostic plus global : «Le cadre temporel restreint des institutions modernes empêche la confiance informelle de mûrir. " Selon cette interprétation, la flexibilité n'ébranle non seulement les habitudes et comportements routiniers, mais agit aussi sur les processus de socialisation qui concernent l'engagement mutuel, la confiance et les expériences qui fondent les jugements des acteurs.

Le principe de la modulation flexible des horaires, en fonction des fluctuations du marché, explique pourquoi la flexibilité du temps de travail s'organise rarement autour des souhaits en temps libre des salariés. Pour une ouvrière de l'automobile, il est en effet difficile d'obtenir des jours de congé compensatoires au printemps, lorsque la production atteint son maximum. De même, il n'est pas facile d'accorder des rencontres amicales, familiales ou associatives pour un ingénieur de recherche quand une réunion de projet peut être convoquée à tout moment ou lorsque le temps de préavis pour un service supplémentaire est de deux jours.

Dans les considérations sociologiques d'Adorno, on trouve un passage qui permet de penser, comment l'insécurité sociale et la peur corrodent les structures de communication horizontale entre salariés ou citoyens, au bénéfice d'un rapport individuel atomisé envers le pouvoir. Le sociologue critique affirme: «Comme les gens ont peur - les chomeurs mais aussi les gens en place qui peuvent à tout moment s'attirer les foudres des investisseurs dont ils représentent les intérêts - ils croient qu'à force de sympathie, d'empressement, de serviabilité et qu'en multipliant les ruses et les détours, en somme grâce à leurs qualités de commerçants, ils pourront se recommander auprès d'un exécutif qu'ils s'imaginent comme omniprésent. ${ }^{1} \gg$.

Adorno W., Theodor (2001), Minima Moralia. Réflexions sur la vie mutilée, Payot, p.20. 


\section{La réponse institutionnelle : flexicurité, pacte pour la compétitivité, agendas sécuritaires}

Les réponses institutionnelles aux processus qui caractérisent l'insécurité sociale varient fortement en fonction des politiques nationales, mais elles convergent néanmoins vers de grands objectifs sur le plan européen, au sujet de la flexibilité du travail et des dispositifs sécuritaires à l'encontre de différentes formes de violence. Le premier aspect a été désigné à travers le terme peu littéraire de "flexicurity» par la commission de l'Union européenne, le second répond aux différents agendas sécuritaires de la commission. Ces deux grands programmes sont explicitement maintenus dans le cadre de la crise économique actuelle.

La «flexicurité » cherche à combiner une ample flexibilité des horaires, des modes de gestion internes aux entreprises et des marchés du travail européen, avec des garanties collectives des salariés. Ce projet ne présente pour le moment qu'un cohérence discursive, en dépit des avancées factuelles de la flexibilité et de la précarité que nous avons soulignées.

Dans le Green paper de la Commission européenne dont il s'agit ici, la création d'un marché du travail flexible est présenté comme le défi majeur de la politique commune ${ }^{1}$. Le document souligne que l'ensemble des formes d'emploi qui ne répondent plus au standard du travail à durée indéterminée et à plein temps sont en progression constante. La Commission part du constat que les contrats et gestions flexibles du travail sont un important facteur de productivité, donc de compétitivité pour les entreprises européennes, qu'il s'agirait de prolonger. Dans ce cadre, elle recommande la généralisation et la pérennisation de ces pratiques, à travers des négociations

\footnotetext{
Commission of the European Communities (11/2006), Green Paper : Modernising labor law to meet the
} challenges of the 21th century, Brussels, p. 7 
collectives. En complément de tels accords, les Etats nationaux sont appelés à prendre des mesures incitatives, sur le plan légal. L'une des questions qu'elle pose aux gouvernements nationaux est de savoir comment « l'adaptation du cadre légal et des accords collectifs peut favoriser la flexibilité et la sécurité de l'emploi ?» (ibidem). La Commission européenne admet cependant que le risque existe de voir des salariés s'enfoncer dans une situation précaire durable, faite de contrats courts et de « petits boulots ». La solution qu'elle voudrait apporter est la mise en place d'un dispositif qui facilite le passage d'un emploi à un autre (la « transition »), avec l'aide des agences pour l'emploi et des institutions de sécurité sociale des Etats membres. Il s'agit de la « flexicurité », puisque les salariés touchés par l'emploi flexible et précaire bénéficieront d'un minimum de droits et d'accompagnement pendant les périodes de non-emploi. Dans ce contexte, la Commission juge que la distinction qu'opère le droit du travail, entre travailleurs indépendants et dépendants, n'est plus opérante. De plus, elle annonce des mesures en faveur de la mobilité et de l'allongement légal du temps de travail, comme la directive en cours de discussion au parlement européen en 2011.

Le dispositif de la flexicurité est généralement accepté par les grands acteurs européens, c'est-à-dire les gouvernements, les principaux partis et syndicats, qui se contentent de proposer des inflexions, des amendements, des compléments. L'un des exemples de cette critique constructive est la suggestion qui émane de la fondation scientifique de la Confédération syndicale allemande, le $\mathrm{DGB}^{1}$. L'auteur propose d'élargir les domaines de la sécurité sociale afin d'encadrer les dispositifs de transition d'un emploi vers l'autre. En France, cette idée est connue sous le terme de « sécurité sociale professionnelle. »

À notre sens, le jeu avec les termes et les mots n'efface pas une opposition de fond, entre un système de flexibilité qui accompagne la demande contractuelle du marché européen, et celui qui voudrait au contraire l'encadrer à travers des organismes de l'Etat. Ainsi, les promoteurs de la

\footnotetext{
1 Tangian, Andranik (2007), Flexibility - Flexicurity - Flexinsurance. Response to the European Commissions Green
} Paper, WSI, Düsseldorf. 
sécurité sociale professionnelle expliquent que ce modèle pourrait fonctionner selon le modèle de la mobilité interne à la fonction publique française, qui permet des passages d'un poste à l'autre, des congés prolongés, des conversions de poste, etc. Ce principe est hérité du jeu des grades qui fut établi lors de la formation de l'Armée française sous Napoléon. L'idée paraît réalisable d'un point de vue strictement logique, mais elle ne tient aucunement compte de la réalité du projet européen tel que nous venons de l'exposer.

\section{Une hypothèse critique}

Pour finir, nous allons lancer une hypothèse sociologique qui ne s'inscrit pas dans ces scénarios, mais qui voudrait éclairer le processus global de la précarisation sociale en Europe, que la flexicurité n'annule en rien comme nous venons de le voir.

La situation empirique nous laisse penser que la flexibilisation et la précarisation, désormais en voie de généralisation, ont atteint un point critique dans la mesure où la majorité des citoyens se sentent désormais directement concernés par ces phénomènes. Il paraît alors possible de rapprocher l'expérience vécue des publics fragilisés par l'insécurité sociale et leur comportement public ou politique (termes rigoureusement identiques selon Habermas).

Analogie troublante, les mêmes publics qui sont particulièrement concernés par l'insécurité sociale, sont aussi frappés par l'absentéisme électoral, le vote protestataire ou le vote sanction (trois variations de la désaffiliation publique). Cela est apparu lors des trois référenda successifs qui ont abouti au rejet des traités européens supposés organiser le cadre politique de l'UE, en France, aux Pays-Bas et en Irlande, entre 2005 et 2008, ainsi qu'aux élections européennes de 2009, dans tout l'espace de 1'UE. Prélude aux contestations de rue massives qui ont suivi en Islande, en Irlande, en Grèce, en France, en Grande Bretagne, en Espagne et au Portugal de 2009 à 2011, sans parler des 
crises politiques à répétition que traversent la Belgique, l'Italie et plusieurs pays d'Europe de l'Est (par exemple la Pologne et la République tchèque).

En France, ce lien peut être retracé de façon nette, puisque les mêmes catégories qui ont provoqué la crise politique des présidentielles de 2002, avec la présence d'un candidat d'extrême droite au second tour, ont aussi majoritairement rejeté le traité constitutionnel européen en 2005. Chômeurs, classes populaires touchant des revenus inférieurs au salaire minimum, ouvriers et employés faiblement qualifiés ont tous voté en nombre contre le traité, selon les enquêtes disponibles. ${ }^{1}$ Par ailleurs, le mouvement social contre le CPE et le CNE, que les législateurs ont explicitement présenté comme une adaptation française de la flexicurité, a mobilisé jusqu'à trois millions de manifestants au printemps 2006, à l'encontre du principe d'insécurité que faisait peser sur les salariés concernés la possibilité d'un licenciement facilité.

En Allemagne, les manifestations de masse contre la flexicurité (lois Hartz) de 2004 ont abouti à la percée d'un parti de gauche contestataire qui, depuis 2006, interdit la formation de coalitions politiques cohérentes, sur le plan national et régional. Les Pays-Bas ont connu une situation similaire.

Nous ne sommes pas en mesure, à cet endroit, de démontrer de façon statistique fiable la coïncidence entre l'expérience de l'insécurité sociale et du rejet majoritaire du projet de l'Union européenne, confirmé par trois référenda successifs et un ensemble de sondages, mais nous venons de livrer un ensemble d'indices qui justifient la formulation d'une telle hypothèse. En revanche, des enquêtes nationales accréditent cette piste. Le rapport de recherche d'une vaste enquête sociologique menée par l'Institut de science politique de Berlin - Oswald Suhr Institut - parmi 4000

$1 \quad$ Cautres/Mayer (2004), Le grand désordre électoral, Presses de la fondation des presses de science politique, Pais ; Kouvélakis, Statis (2007), Les luttes sociales en France, Textuel, Paris. 
salariés allemands montre la correspondance entre la précarisation de l'emploi d'un côté et la disponibilité d'un cinquième des salariés pour des solutions autoritaires ou nationalistes ${ }^{1}$, ce qui déstabilise la représentativité traditionnelle des syndicats et partis. Concernant l'Europe de l'Est, une corrélation similaire a été établie récemment par Attila Agh : «La réalité sociologique la plus élémentaire montre qu'une nouvelle underclass large s'est manifestée en Europe de l'Est sur le plan social et politique et ce fait intervient dans les mobilisations des perdants, qui prennent la forme d'alliances aussi hétérogènes que larges ${ }^{2}$. »

Dès lors, le dispositif de flexicurité, de même que les agendas sécuritaires destinés à prévenir la violence criminelle, terroriste ou encore les troubles à l'ordre public, remplissent avant tout une fonction discursive, susceptible de compenser la désaffiliation publique des publics touchés par l'insécurité sociale à travers une représentation institutionnelle rassurante. Pour le moment, cette représentation publique a toutes les difficultés d'organiser un espace public qui serait susceptible d'accueillir les expériences qualitatives de l'insécurité et de favoriser leur verbalisation.

Zeuner, Bodo (2007), Gewerkschaften und Rechtsextremismus, Westfälisches Dampfboot, Münster ; Neumann, Alexander (2008), Peur, bruits, odeurs. La personnalité autoritaire face aux dispositifs sécuritaires, Mouvements N.55, La Découverte., Paris

2 Agh, Attila, (2007) « Post-accession crisis and social challenge in ECE », in : Agh/Ferencz, Overcoming the EU crisis, Together for Europe Research Center, Budapest. 
La vie mutilée des citoyens risque ainsi d'entraver le projet européen lui-même, qui bute sur les effets déstabilisants qui sont provoqués par sa propre méthode de construction. Les expressions publiques qui voient le jour à la faveur de la crise mondiale - grèves locales non encadrés, grèves générales couvertes par les syndicats, mouvements contestataires ou insurrectionnels de la jeunesse, vote sanction et vote protestataire - nous invitent à rétablir un dialogue entre la sociologie du travail, les études socio-psychologiques et la sociologie politique, qui fut interrompu à la fin des années 70.

\begin{abstract}
Alexander Neumann
Cet article s'appuie sur un working-paper au sein du projet de recherche intégré du 6ème programme du département de recherche de l'Union européenne, Challenge, et dont la première version fut rédigée en 2008 .
\end{abstract}

